\title{
An Environmental Science and Engineering Framework for Combating Antimicrobial Resistance
}

\author{
Amy Pruden, ${ }^{1, *, \dagger}$ Reinaldo E. Alcalde, Pedro J.J. Alvarez, ${ }^{3}$ Nicholas Ashbolt, ${ }^{4}$ Heather Bischel, \\ Natalie L. Capiro, Emily Crossette, Dominic Frigon, ${ }^{8}$ Kassandra Grimes, ${ }^{9}$ Charles N. Haas, ${ }^{10}$ \\ Kaoru Ikuma, ${ }^{11}$ Anthony Kappell, ${ }^{12}$ Timothy LaPara, ${ }^{13}$ Lee Kimbell, ${ }^{12}$ Mengyan Li, ${ }^{14}$ Xu Li, ${ }^{15}$ \\ Patrick McNamara, ${ }^{12}$ Youngwoo Seo, ${ }^{16}$ Mark D. Sobsey, ${ }^{17}$ Emanuele Sozzi, ${ }^{17}$ \\ Tala Navab-Daneshmand, ${ }^{18}$ Lutgarde Raskin, Maria Virginia Riquelme, \\ Peter Vikesland, Krista Wigginton, and Zhi Zhou ${ }^{19}$ \\ ${ }^{1}$ Department of Civil \& Environmental Engineering, Virginia Tech, Blacksburg, Virginia. \\ ${ }^{2}$ Department of Civil, Architectural, \& Environmental Engineering, University of Texas, Austin, Texas. \\ ${ }^{3}$ Department of Civil \& Environmental Engineering, Rice University, Houston, Texas. \\ ${ }^{4}$ School of Public Health, University of Alberta, Edmonton, Alberta, Canada. \\ ${ }^{5}$ Department of Civil \& Environmental Engineering, University of California, Davis, California. \\ ${ }^{6}$ Department of Civil \& Environmental Engineering, Tufts University, Medford, Massachusetts. \\ ${ }^{7}$ Department of Civil \& Environmental Engineering, University of Michigan, Ann Arbor, Michigan. \\ ${ }^{8}$ McGill University, Montreal, Quebec, Canada. \\ ${ }^{9}$ Department of Civil \& Environmental Engineering, University of Virginia, Charlottesville, Virginia. \\ ${ }^{10}$ Department of Civil, Architectural, \& Environmental Engineering, Drexel University, Philadelphia, Pennsylvania. \\ ${ }^{11}$ Department of Civil, Construction, \& Environmental Engineering, lowa State University, Ames, lowa. \\ ${ }^{12}$ Department of Civil, Construction, \& Environmental Engineering, Marquette University, Milwaukee, Wisconsin. \\ ${ }^{13}$ Department of Civil, Environmental, and Geo-Engineering, University of Minnesota, Minneapolis, Minnesota. \\ ${ }^{14}$ Department of Chemistry \& Environmental Sciences, New Jersey Institute of Technology, University Heights Newark, New Jersey. \\ ${ }^{15}$ Department of Civil Engineering, University of Nebraska, Lincoln, Nebraska. \\ ${ }^{16}$ Department of Civil \& Environmental Engineering, University of Toledo, Toledo, Ohio. \\ ${ }^{17}$ Gillings School of Public Health, University of North Carolina, Chapel Hill, North Carolina. \\ ${ }^{18}$ Department of Chemical, Biological, and Environmental Engineering, Oregon State University, Corvalis, Oregon. \\ ${ }^{19}$ Department of Environmental and Ecological Engineering and Civil Engineering, Purdue University, West Lafayette, Indiana.
}

Received: December 14, 2017

Accepted in revised form: April 6, 2018

\begin{abstract}
On June 20, 2017, members of the environmental engineering and science (EES) community convened at the Association of Environmental Engineering and Science Professors (AEESP) Biennial Conference for a workshop on antimicrobial resistance. With over 80 registered participants, discussion groups focused on the following topics: risk assessment, monitoring, wastewater treatment, agricultural systems, and synergies. In this study, we summarize the consensus among the workshop participants regarding the role of the EES community in understanding and mitigating the spread of antibiotic resistance via environmental pathways. Environmental scientists and engineers offer a unique and interdisciplinary perspective and expertise needed for engaging with other disciplines such as medicine, agriculture, and public health to effectively address important knowledge gaps with respect to the linkages between human activities, impacts to the environment, and human health risks. Recommendations that propose priorities for research within the EES community, as well as areas where interdisciplinary perspectives are needed, are highlighted. In particular, risk modeling and assessment, monitoring, and mass balance modeling can aid in the identification of "hot spots" for antibiotic resistance evolution and dissemination, and can help identify effective targets for mitigation. Such information will be essential for the development of an informed and effective policy aimed at preserving and protecting the efficacy of antibiotics for future generations.
\end{abstract}

*Corresponding author: Department of Civil \& Environmental Engineering, Virginia Tech, 418 Durham Hall, Virginia Tech, Blacksburg, VA 24060. Phone: +1 (540) 231-3980; Fax: (540) 231-

7916; E-mail: apruden@vt.edu

${ }^{\dagger}$ Member of AEESP. 
Keywords: biological systems; environmental microbiology; molecular biology; monitoring methods; risk

\section{Key Knowledge Gaps for Antibiotic Resistance in the Environment and Research Needs}

$\mathbf{M}$

ASS PRODUCTION AND USE OF ANTIMICROBIALS, especially antibiotics aimed specifically at treating and preventing bacterial infections, represent a two-edged sword. They provide revolutionary public health benefits, but overuse is linked to diminishing returns because of the potential to select for resistant strains. The growing global threat of antibiotic resistance to human health is widely acknowledged. The following subsections summarize the key knowledge gaps and research needs for better understanding and mitigating environmental sources and pathways of antibiotic resistance in working toward preserving the utility of these life-saving drugs.

\section{Risk modeling and assessment}

Substantial advances have recently been made in the field of quantitative microbial risk assessment (QMRA). These advances provide a framework for determining concentrations of pathogens via various environmental exposure routes that are capable of causing illness (Haas et al., 2014; Haas, 2015). However, there is an urgent need to extend the QMRA concept in a way that captures the environmental dimension of antibiotic resistance (Ashbolt et al., 2013). Progress toward risk assessment is essential to link human exposure to antibiotic-resistant bacteria (ARBs) able to infect humans and antibiotic resistance genes (ARGs) present in the environment or excreted by domestic animals or humans.

Identifying critical risk thresholds for ARB and ARG exposures through food, water, and air that influence human health will be particularly key to develop mitigation strategies (Vikesland et al., 2017). For traditional pathogens, such information can be derived from dose-response studies; however, antibiotic resistance requires a different paradigm. For example, instances of multiple exposures to bacteria carrying ARGs, asymptomatic colonization that may be followed by delayed onset of illness with an AMR organism, multiple interacting/competing strains of susceptible/resistant pathogens, and horizontal gene transfer in the environment and/or host complicate the traditional dose-response paradigm (Wassenaar et al., 2007; Evans et al., 2009; Garner et al., 2016) and are likely to be important and challenging for risk assessment. While the most meaningful monitoring targets are yet to be identified, methods that provide quantitative measures of exposure and health effects will provide the greatest value from a risk characterization and assessment standpoint. Epidemiological studies that document the extent of acquisition of AMR organisms from environmental exposures or that quantify the acquisition of such bacteria from human or animal dose-response studies would be of value.

As a first step, a source, fate, and transport-based framework are logical means to capture the complexity of antibiotic resistance movement in the environment and to identify human exposure "hot spots" as well as points of targeted intervention. Such a framework, where animals, humans, and the "environment" represent three reservoirs or "stocks" of antibiotic resistance between which there are bidirectional transports or "flows" (Fig. 1), could be adapted from prior work (Haas, 1983, 2000; Rose and Haas, 1999; Pujol et al., 2009; Haas et al., 2014). Example flows between compartments include pharmaceutical wastewater discharges to receiving waters, carriage of pathogenic bacteria or other biota carrying ARGs on meat or produce, classic exposure routes to human and animal excreta through air, water, and soil, irrigation with contaminated water, and application of manure or biosolid-derived soil amendments. The configuration and relative impact of each of these compartments may differ substantially in more developed and resource-rich countries where there is more rigorous implementation and enforcement of sanitation barriers, in contrast to countries where barriers between anthropogenic outputs and the environment as well as other sanitation strategies may be limited or nonexistent.

Complex dynamics within each reservoir, largely governed by microbial ecological processes, determines the prevailing levels of resistance. For example, the physicochemical conditions (e.g., concentration of antimicrobials, metals, or other stressors) and biotic factors (e.g., presence of mobile genetic elements [MGEs]) may interact in the selection of resistant versus susceptible strains. Importantly, in the absence of antibiotics, the actual competitive advantage of resistant strains relative to susceptible strains is currently unclear. In this context, the perspective from which the environmental engineering and science (EES) community defines "antimicrobial resistance" may play a role in the resolution at which risk is defined. For example, defining antibiotic resistance from a broad perspective (e.g., as one composite contaminant) could aid in identifying key environmental hot spots as well as in comparing their relative significance in terms of overall risk. On the contrary, a deeper look that separates ARGs into health risk classifications, such as those proposed by Martínez et al. (2015), within an environmental context, could yield higher resolution models that lead to more specific recommendations.

Coupling environmental monitoring of exposure sources with human epidemiological studies, for example, studies involving livestock farm workers and wastewater treatment plant (WWTP) operators (Rosenberg Goldstein et al., 2014), could be particularly informative toward advancing exposure monitoring, health effects analysis, and risk assessment. Risk assessment coupled with surveillance monitoring could guide the development of fruitful strategies for mitigating the spread of antibiotic resistance (Pruden et al., 2013). For example, modeling and sensitivity analysis can help identify which parameters are most influential, and thus, the most promising targets for control in environmental systems.

\section{Surveillance monitoring}

International institutions such as the World Health Organization (WHO), the European Union health organizations (EU Commission, 2017) and the nations adhering to the Joint Programming Initiative on Antimicrobial Resistance (JPIAMR, 2017), strongly advocate the need for increased and coordinated monitoring of antibiotic resistance (WHO, 2014a, 2015; Wuijts et al., 2017). Monitoring characterizes the landscape of antibiotic resistance, identifies key routes of 


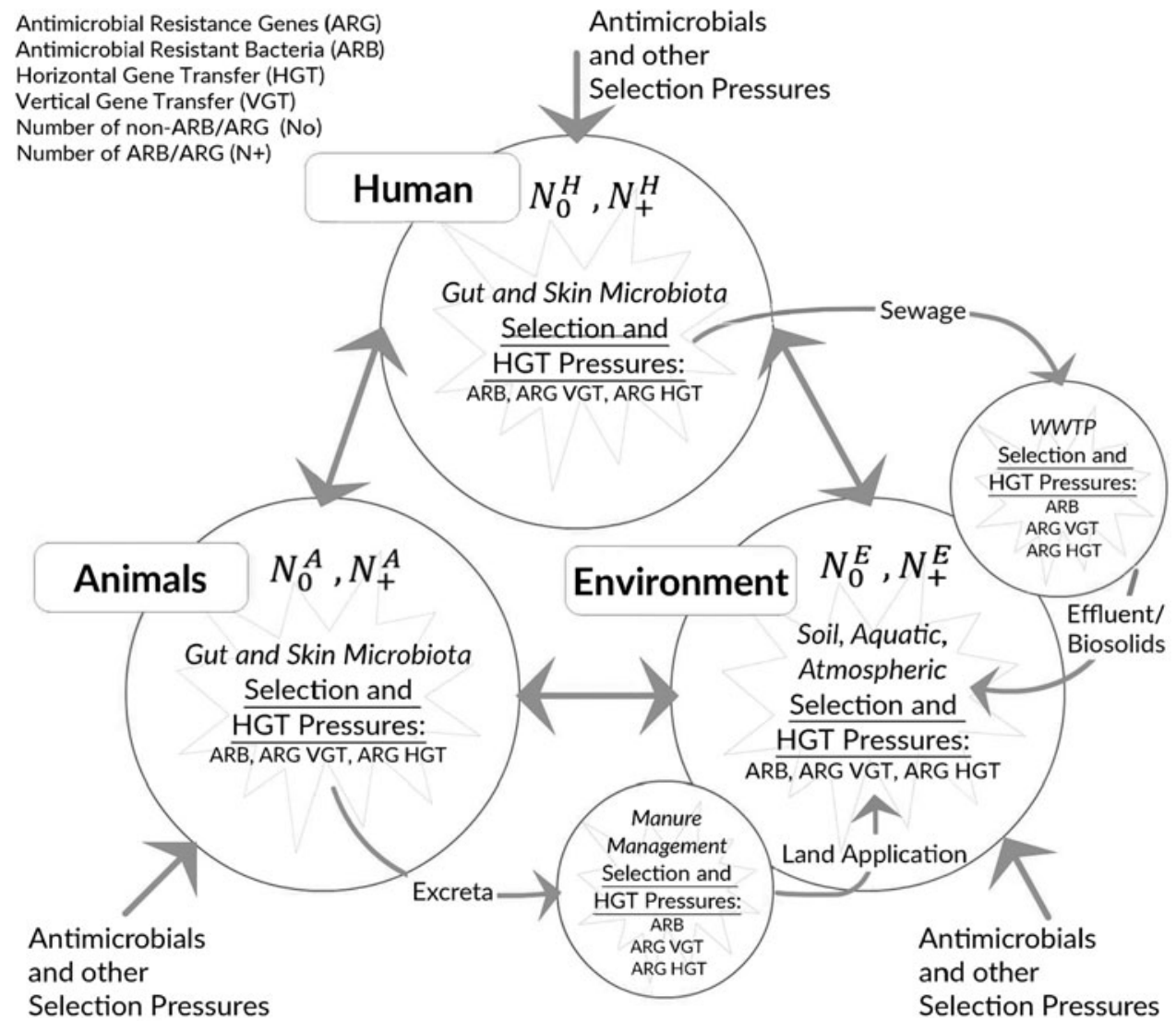

FIG. 1. Stocks and Flows Framework Conceptualizing the Movement of Antimicrobial Resistance in the Environment. $\mathrm{N}_{0}$ is defined as the total number of bacteria sensitive to antibiotics and other selective pressures. $\mathrm{N}+$ is defined as the total number of bacteria resistant to antibiotics and other selective pressures. $\mathrm{N}_{0}$ and $\mathrm{N}+$ are a function of growth rates (i.e., $\left.\mu_{0}, \mu+\right)$ to be applied in fate and transport models. exposure, and informs where to most effectively place barriers to the spread of resistance. However, the lack of agreedupon environmental monitoring targets is a major challenge. One potential approach is to adapt standard fecal bacterial monitoring, which forms much of the global regulatory fabric, but focus specifically on resistant strains and genes of concern. For example, the WHO, the EU, and select countries in Asia and Africa have recently initiated a pilot surveillance program targeting extended-spectrum beta lactamase (ESBL)-producing Escherichia coli (Matheu et al., 2017). The spread of ESBL is of utmost concern as a human health threat, whereas monitoring capabilities for E. coli are readily available and accessible worldwide. Other culture-based targets are also worthy of consideration, and it may be advisable at a minimum to culture representative gram-positive and gram-negative indicator bacteria, since antibiotic classes generally vary in their effectiveness toward these two groups. Culturing verifies the viability of target organisms and standardized assays (e.g., CLSI) and can be used to classify resistant strains (McLain et al., 2016). However, viable but nonculturable strains, similarly important to human health risks, will not be captured.

It is important to recognize that culturing misses the majority of bacteria and fails to fully capture the ecological processes driving the dynamics within and between the various reservoirs of resistance (Fig. 1). In this study, nucleic acid-based methods such as next-generation DNA sequencing, metagenomics, and quantitative polymerase chain reaction (qPCR), provide the capacity for monitoring ARG movement within and between environmental compartments. In addition, molecular monitoring can provide data to iden- tify the mechanisms contributing to the spread of antibiotic resistance, such as selection pressures, horizontal gene transfer, or transport of bacteria carrying ARGs. Knowing such mechanisms can help inform risk assessments and lead to more effective mitigation (Luby et al., 2016).

Even as the majority of ARGs and MGEs can be captured with emerging metagenomic capabilities (i.e., the resistome) (Wright, 2007), it remains necessary to accurately identify them and appropriately analyze and interpret the data (Bengtsson-Palme et al., 2017; Lakin et al., 2017). Analogous to the fecal indicator paradigm, it would be useful to identify meaningful ARG and/or MGE targets indicative of AMR potential that could be subject to standardized monitoring. When selecting suitable ARG targets, important considerations include whether the form of resistance an ARG encodes is relevant to human health, if the ARG targets are associated with MGEs and have the capability to spread among different types of bacteria, if the ARG is present inside a human pathogen, and if the ARG is functional or deactivated (e.g., following disinfection) (Martínez et al., 2015).

Importantly, even after cellular deactivation, extracellular DNA may remain functional with the potential to be taken up by other bacteria via transformation (Allen et al., 2010; Dodd, 2012). Furthermore, it is essential that the EES community move toward standards in reporting, such as the types and methods of DNA extraction used and any QA/QC, detection limits, specificity, providing data in both normalized and un-normalized formats, sequencing depths, and criteria and cutoffs for identifying an ARG or MGE. Studies must be reported in a manner that is repeatable. In addition, 
Table 1. Recommended Priorities for Research and Action Among the EES Community

General area in need of action

Surveillance and monitoring
Recommended priorities for research and/or action

Standardization of methods and QA/QC practices

Agreement on targets for monitoring

Agreement on definition of antibiotic resistance in an environmental context

Curation and structuring of antibiotic resistance databases to include ARG risk classification

Standardization of data reporting (and enforcement within EES community)

AMR characterization within environmental hot spots (e.g., WWTPs, farms, pharmaceutical effluents)

Characterization of mechanisms of selection, transfer, and propagation of antibiotic resistance

Identification of effective treatment technologies for minimization of AR outputs into receiving environments

Expansion of international studies that provide insight into broader phenomena, including measurable effects of various antibiotic regulation and control strategies, hygiene and sanitation conditions, water and agricultural infrastructure, and socioeconomic factors

Risk modeling and assessment
Extension of QMRA to capture environmental dimension of antibiotic resistance

Expansion of interdisciplinary system-level studies that focus on risk characterization

Modeling of antibiotic resistance fate and transport and/or mass balances

Identification of links between environmental exposure and epidemiological data

Quantify links between degree of sanitation implementation and associated antibiotic resistance risks

QMRA, quantitative microbial risk assessment; WWTPs, wastewater treatment plants.

metagenomic methods need to be improved to obtain absolute quantification, rather than just relative abundance.

\section{Wastewater treatment}

WWTPs, given their high bacterial densities and that they receive antibiotics, metals, and other selective agents, are a logical focal point of the EES community. Numerous studies have now profiled and tracked ARGs and ARBs through municipal WWTPs, as well as the impacts of effluent and biosolids on their receiving environments, but there is a need for translation of findings into practical, meaningful, and actionable guidance for WWTP designers and operators. Future studies should inform quantitative estimates of relative loadings from solid versus liquid product streams as a guide for where to focus attention for mitigation (Munir et al., 2011) and to provide context with respect to WWTP-associated loadings relative to other potential sources. A key challenge is to identify if there are critical AMR and/or selective agent loading rates that may lead to measurable concentration increases or decreases, to inform possible control targets. Also, it is essential for wastewater management strategies to be relevant to local conditions. For example, cost-effective and lowtechnology solutions are particularly critical in lesser developed countries. Ensuring basic sanitation as the primary barrier for AMR spread should be the first and foremost goal of any antibiotic resistance management action plan (Laxminarayan, 2014; Andremont and Walsh, 2015).

A clear quantitative analysis of the ecological processes occurring within biological treatment systems is needed to assess their net contribution toward attenuating (e.g., reducing fitness of resistance strains) or propagating (e.g., providing an environment ideal for selection and horizontal gene transfer) antibiotic resistance. Furthermore, moving beyond observational studies toward more controlled, mechanistic studies that may better identify factors that promote or diminish antibiotic resistance transfer during biological treatment is necessary. Of interest are the relative roles of antibiotics, metals, virulence factors, and resistant bacteria and pathogens in the influent and effluent, versus operational conditions such as suspended or attached growth, aerobic versus anaerobic, mesophilic versus thermophilic, solids retention time, and hydraulic residence times (Diehl and LaPara, 2010; Zhang and Li, 2011; Bengtsson-Palme et al., 2016; Neyestani et al., 2017a, 2017b). Studies that address physiology, ecology, and processes for managing the microbial ecology of AMR during wastewater treatment are needed, along with prioritization of waste streams with high potential to spread AMR, such as those from antibiotic production processes and hospitals.

A multibarrier approach to wastewater treatment may be most advisable to minimize the impacts of ARBs and ARGs from the application of residual biosolids as a soil amendment and in wastewater reuse. For biosolids, hydrolytic treatments, anaerobic digestion (particularly thermophilic), and composting are potential options (Diehl and LaPara, 2010; Zhang and Li, 2011; Bengtsson-Palme et al., 2016). Ideally, effluent treatment strategies should disinfect and destroy ARGs and remove potential selecting agents such as residual antibiotics or metals (Dodd, 2012). For the final effluent, membrane-based treatments will likely offer the most stringent treatment. Although several studies have addressed the efficacy of disinfection strategies against antibiotic resistance (McKinney and Pruden, 2012; Czekalski et al., 2016; Chang et al., 2017), currently, surprisingly little is 
known about potential unintended effects of various disinfectants, including chlorine, chloramines, ozone, and other advanced oxidation processes, other than the possibility that they select for more resistant (and multidrug-resistant) strains (Luo et al., 2014; Guo et al., 2015). Some studies have identified circumstances that select for resistant strains, which then have the opportunity to preferentially regrow downstream (Xi et al., 2009). This observation highlights the need to consider monitoring environmental pathogens (e.g., Aeromonas or Arcobacter spp.) that grow during and following treatment (Banting et al., 2016; Rasmussen-Ivey et al., 2016). Overall, mitigation strategies must be harmonized with the need for water sustainability and reuse, because the societal need for water overrides the possible risks of antibiotic resistance.

\section{Agriculture}

Antibiotics are widely used in the livestock industry, more so on a mass basis than in humans (Van Boeckel et al., 2015). The EU phased out subtherapeutic use of antibiotics over a decade ago, while the United States only recently followed suit (December 2016) with the Veterinary Feed Directive requiring a prescription for antibiotic use in livestock and prohibiting the use of antibiotics as growth promoters (FDA, 2012, 2013, 2017). More recently, in 2017, the WHO published its "Guidelines on use of medically important antimicrobials in food-producing animals" following the 5th revised list of "critically important antimicrobials for human medicine", published in 2014 (WHO, 2014b, 2017). An interdisciplinary system perspective along the full "farm to fork" continuum is recommended to investigate the impacts of these policies.

Collaborating with and learning from the food and agricultural sciences can aid in identifying promising on-farm mitigation opportunities, including manure management, pre- and postharvest practices, and meat processing and packing. With this approach, there are clear synergies with current calls to advance the efficiency of food-energy-water systems. System complexity and nonpoint contributions are major challenges to address. As with wastewater treatment, multiple barriers are advisable (O'Neill et al., 2015), especially given the complexity of nonpoint source mitigation. In contrast to the management and treatment of municipal wastewater, food animal waste management has no regulated fecal microbial reduction requirements in the United States and most other countries. Developing effective and economical approaches to minimize ARBs and ARGs in animal manure is a critical challenge.

Key issues in recommending mitigation strategies for agricultural sources of antibiotic resistance include the following. Are current guidelines and policies effectively implemented in practice and spirit, if so, are they working beneficially with respect to both animal health and environmental conservation, and what are the synergies with other drivers such as consumer preference and the "organic" movement? There is an opportunity to learn from Europe and other countries regarding successful interventions. Furthermore, attenuation of resistance may require decades to be observable, particularly if resistant bacteria have acquired a permanent niche within the livestock microbiome. Environmental modeling could help predict the effects of changes in antibiotic use policies and mitigation options. Recognizing that farmers are typically operating with marginal profits makes it critical that recommendations do not put people out of business and hurt the food supply in years to come, especially as population growth and food demands become increasingly acute.

\section{Recommendations}

Environmental scientists and engineers provide a unique and valuable perspective and tool set toward understanding and addressing antibiotic resistance. Recommendations regarding priority areas where the EES community may contribute toward countering the spread of antibiotic resistance via environmental pathways are highlighted in Table 1. In particular, large interdisciplinary system-level studies that focus on risk characterization, especially those grounded in real-world conditions and practice, can provide AMR monitoring and yield recommendations for mitigation strategies in environmental systems. Such studies could ideally be coupled with fate and transport/mass balance modeling, using a core analytical system framework that is well established in the EES community.

In particular, hypothesis-driven environmental research that provides insight into mechanisms of selection, transfer, and propagation of antibiotic resistance and its linkage to human health risk assessments are needed. Development of risk assessment models specifically tailored to the environmental roles of antibiotic resistance was identified as a top priority, especially given the need for actionable endpoints for both monitoring and mitigation. International studies are also of value, providing insight into broader phenomena, including measurable effects of various antibiotic regulation and control strategies, hygiene and sanitation conditions, water and agricultural infrastructure, and socioeconomic factors. There is a need for rigorous QA/QC in data collection, as well as agreement in the community regarding standardized methods and reporting. Until priority monitoring targets are agreed on, analysis of a suite of culture-based and molecularbased indicators is logical, as is making raw data and unit analysis publicly available. Such criteria should be considered in the peer-reviewed evaluation of literature.

Collectively, contribution from the EES community is essential to inform effective practices and policies for combatting antibiotic resistance toward preserving and protecting antibiotics for continued benefit and protection of public health in the future.

\section{Acknowledgments}

The authors thank and acknowledge all the participants in the Slowing the Spread of Antimicrobial Resistance via Environmental Pathways: Risk Assessment and Management Perspective Workshop that was held at the Association of Environmental Engineering and Science Professors (AEESP) Biennial Conference, June 20, 2017, University of Michigan, Ann Arbor, MI, as well as the National Science Foundation Partnership in International Research and Education Award 1545756. Participants representing each of the subgroup topics volunteered to synthesize the discussions and contribute as coauthors to this article.

\section{Author Disclosure Statement}

No competing financial interests exist. 


\section{References}

Allen, H.K., Donato, J., Wang, H.H., Cloud-Hansen, K.A., Davies, J., and Handelsman, J. (2010). Call of the wild: Antibiotic resistance genes in natural environments. Nat. Rev. Microbiol. 8, 251.

Andremont, A., and Walsh, T. (2015). The role of sanitation in the development and spread of antimicrobial resistance. $A M R$ Control. 67.

Ashbolt, N.J., Amezquita, A., Backhaus, T., Borriello, P., Brandt, K.K., Collignon, P., Coors, A., Finley, R., Gaze, W.H., Heberer, T., Lawrence, J.R., Larsson, D.G., McEwen, S.A., Ryan, J.J., Schonfeld, J., Silley, P., Snape, J.R., Van den Eede, C., and Topp, E. (2013). Human Health Risk Assessment (HHRA) for environmental development and transfer of antibiotic resistance. Environ. Health Perspect. 121, 993.

Banting, G.S., Braithwaite, S., Scott, C., Kim, J., Jeon, B., Ashbolt, N., Ruecker, N., Tymensen, L., Charest, J., Pintar, K., Checkley, S., and Neumann, N.F. (2016). Evaluation of Various Campylobacter-Specific Quantitative PCR (qPCR) Assays for Detection and Enumeration of Campylobacteraceae in Irrigation Water and Wastewater via a Miniaturized Most-Probable-Number-qPCR Assay. Appl. Environ. Microbiol. 82, 4743.

Bengtsson-Palme, J., Hammaren, R., Pal, C., Ostman, M., Bjorlenius, B., Flach, C.F., Fick, J., Kristiansson, E., Tysklind, M., and Larsson, D.G.J. (2016). Elucidating selection processes for antibiotic resistance in sewage treatment plants using metagenomics. Sci. Total Environ. 572, 697.

Bengtsson-Palme, J., Larsson, D.G.J., and Kristiansson, E. (2017). Using metagenomics to investigate human and environmental resistomes. J. Antimicrob. Chemother. 72, 2690.

Chang, P.H., Juhrend, B., Olson, T.M., Marrs, C.F., and Wigginton, K.R. (2017). Degradation of extracellular antibiotic resistance genes with UV254 treatment. Environ. Sci. Technol. 51, 6185.

Czekalski, N., Imminger, S., Salhi, E., Veljkovic, M., Kleffel, K., Drissner, D., Hammes, F., Burgmann, H., and von Gunten, U. (2016). Inactivation of antibiotic resistant bacteria and resistance genes by ozone: from laboratory experiments to fullscale wastewater treatment. Environ. Sci. Technol. 50, 11862.

Diehl, D.L., and LaPara, T.M. (2010). Effect of temperature on the fate of genes encoding tetracycline resistance and the integrase of class 1 integrons within anaerobic and aerobic digesters treating municipal wastewater solids. Environ. Sci. Technol. 44, 9128.

Dodd, M.C. (2012). Potential impacts of disinfection processes on elimination and deactivation of antibiotic resistance genes during water and wastewater treatment. J. Environ. Monit. 14, 1754.

EU_Commission. (2017). A European One Health Action Plan against Antimicrobial Resistance (AMR). Bruxelles: EU.

Evans, M.R., Northey, G., Sarvotham, T.S., Rigby, C.J., Hopkins, A.L., and Thomas, D.R. (2009). Short-term and medium-term clinical outcomes of quinolone-resistant Campylobacter infection. Clin. Infect. Dis. 48, 1500.

FDA. (2012). FDA \#209: Guidance for Industry - The Judicious Use of Medically Important Antimicrobial Drugs in FoodProducing Animals, Administration, U.S.D.o.H.a.H.S.-F.a.D., Medicine, C.f.V., eds. Rockville, MD: Center for Veterinary Medicine, Food and Drug Administration.

FDA. (2013). FDA \#213: Guidance for Industry - New Animal Drugs and New Animal Drug Combination Products Ad- ministered in or on Medicated Feed or Drinking Water of Food-Producing Animals: Recommendations for Drug Sponsors for Voluntarily Aligning Product Use Conditions with GFI \#209, Administration, U.S.D.o.H.a.H.S.-F.a.D., Medicine, C.f.V., eds. Rockville, MD: Center for Veterinary Medicine, Food and Drug Administration.

FDA. (2017). FDA FACT SHEET: Veterinary Feed Directive Final Rule and Next Steps, Administration, U.S.D.o.H.a.H.S.F.a.D., Medicine, C.f.V., eds. Rockville, MD: Center for Veterinary Medicine, Food and Drug Administration.

Garner, E., Zhu, N., Strom, L., Edwards, M., and Pruden, A. (2016). A human exposome framework for guiding risk management and holistic assessment of recycled water quality. Environ. Sci. Water Res. Technol. 2, 580.

Guo, M.T., Yuan, Q.B., and Yang, J. (2015). Distinguishing effects of ultraviolet exposure and chlorination on the horizontal transfer of antibiotic resistance genes in municipal wastewater. Environ. Sci. Technol. 49, 5771.

Haas, C.N. (1983). Estimation of risk due to low doses of microorganisms: A comparison of alternative methodologies. Am. J. Epidemiol. 118, 573.

Haas, C.N. (2000). Epidemiology, microbiology, and risk assessment of waterborne pathogens including Cryptosporidium. J. Food Prot. 63, 827.

Haas, C.N. (2015). Microbial dose response modeling: Past, present, and future. Environ. Sci. Technol. 49, 1245.

Haas, C.N., Rose, J.B., and Gerba, C.P. (2014). Quantitative Microbial Risk Assessment, Second edition. Edition. New York: John Wiley \& Sons, Inc., p. cm p.

JPIAMR. (2017). Joint Program Initiative on Antimicrobial Resistance (JPIAMR) 2013 Strategic Research Agenda: Overview, Actions and Potential Needs. Available at: www.jpiamr .eu (accessed May 4, 2018).

Lakin, S.M., Dean, C., Noyes, N.R., Dettenwanger, A., Ross, A.S., Doster, E., Rovira, P., Abdo, Z., Jones, K.L., Ruiz, J., Belk, K.E., Morley, P.S., and Boucher, C. (2017). MEGARes: An antimicrobial resistance database for high throughput sequencing. Nucleic Acids Res. 45, D574.

Laxminarayan, R. (2014). Antibiotic effectiveness: Balancing conservation against innovation. Science. 345, 1299.

Luby, E., Ibekwe, A.M., Zilles, J., and Pruden, A. (2016). Molecular Methods for Assessment of Antibiotic Resistance in Agricultural Ecosystems: Prospects and Challenges. $J$. Environ. Qual. 45, 441.

Luo, Y., Yang, F., Mathieu, J., Mao, D., Wang, Q., and Alvarez, P. (2014). Proliferation of multidrug-resistant New Delhi Metallobeta-lactamase genes in municipal wastewater treatment plants in Northern China. Environ. Sci. Technol. Lett. 1, 26.

Martínez, J.L., Coque, T.M., and Fernando, B. (2015). What is a resistance gene? Ranking risk in resistomes. Nat. Rev. Microbiol. 13, 116.

Matheu, J., Aidara-kane, A., and Andremont, A. (2017). The ESBL Tricycle Antimicrobial Resistance Surveillance Project: A Simple, One Health Approach to Global Surveillance. AMR CONTROL 2017 Edition, pp. 55-58. Available at: http:// resistancecontrol.info/2017/the-esbl-tricycle-amr-surveillanceproject-a-simple-one-health-approach-to-global-surveillance/ (accessed May 4, 2018).

McKinney, C.W., and Pruden, A. (2012). Ultraviolet disinfection of antibiotic resistant bacteria and their antibiotic resistance genes in water and wastewater. Environ. Sci. Technol. 46, 13393.

McLain, J.E., Cytryn, E., Durso, L.M., and Young, S. (2016). Culture-based methods for detection of antibiotic resistance in 
agroecosystems: advantages, challenges, and gaps in knowledge. J. Environ. Qual. 45, 432.

Munir, M., Wong, K., and Xagoraraki, I. (2011). Release of antibiotic resistant bacteria and genes in the effluent and biosolids of five wastewater utilities in Michigan. Water Res. 45,681 .

Neyestani, M., Dickenson, E., McLain, J., Obergh, V., Quinones, O., Rock, C., and Gerrity, D. (2017a). Solids retention time, influent antibiotic concentrations, and temperature as selective pressures for antibiotic resistance in activated sludge systems. Environ. Sci. Water Res. Technol. 3, 383.

Neyestani, M., Dickenson, E., McLain, J., Robleto, E., Rock, C., and Gerrity, D. (2017b). Impacts of solids retention time on trace organic compound attenuation and bacterial resistance to trimethoprim and sulfamethoxazole. Chemosphere. $182,149$.

O’Neill, J., Borriello, P., Davies, S.D., Denning, D., Health, S.a.t.D.o., Farrar, J., Fuglie, K., Gaze, W., Hamied, Y.K., Healey, K., Veterinary Medicines Directorate, D.o.E., Food and Rural Affairs, U., Hollis, A., Johnson, E.M., Laboratory, N.I.S., Public Health England, Kümmerer, K., Resources, L.U., Germany, Larsson, J., Resistance Research, U.o.G., Malakar, P., McAdams, D., Mulaney, B., Murray, F., Ormel, H.J., Outterson, K., Piddock, L., England, S.a.P.H., Rex, J., Ryan, M., Rushton, J., and Salin, K. (2015). Antimicrobials in Agriculture and the Environment: Reducing Unnecessary Use and Waste. Available at: https://amr-review.org/sites/default/ files/Antimicrobials\%20in\%20agriculture\%20and $\% 20$ the $\%$ 20environment $\% 20-\% 20$ Reducing $\% 20$ unnecessary $\% 20$ use $\%$ 20and\%20waste.pdf (accessed May 4, 2018).

Pruden, A., Larsson, D.G., Amezquita, A., Collignon, P., Brandt, K.K., Graham, D.W., Lazorchak, J.M., Suzuki, S., Silley, P., Snape, J.R., Topp, E., Zhang, T., and Zhu, Y.G. (2013). Management options for reducing the release of antibiotics and antibiotic resistance genes to the environment. Environ. Health Perspect. 121, 878.

Pujol, J.M., Eisenberg, J.E., Haas, C.N., and Koopman, J.S. (2009). The effect of ongoing exposure dynamics in dose response relationships. PLoS Comput. Biol. 5, e1000399.

Rasmussen-Ivey, C.R., Figueras, M.J., McGarey, D., and Liles, M.R. (2016). Virulence Factors of Aeromonas hydrophila: In the Wake of Reclassification. Front Microbiol. 7, 1337.
Rose, J.B., and Haas, C.N. (1999). A risk assessment framework for the evaluation of skin infections and the potential impact of antibacterial soap washing. Am. J. Infect. Control. 27, S26. Rosenberg Goldstein, R.E., Micallef, S.A., Gibbs, S.G., He, X., George, A., Sapkota, A., Joseph, S.W., and Sapkota, A.R. (2014). Occupational exposure to Staphylococcus aureus and Enterococcus spp. among spray irrigation workers using reclaimed water. Int. J. Environ. Res. Public Health. 11, 4340.

Van Boeckel, T.P., Browerb Charles, Gilbertc Marius, T. Grenfella Bryan, Levina Simon A., Robinsoni Timothy P., and Teillanta Aude, Ramanan, L. (2015). Global trends in antimicrobial use in food animals. Proc. Natl. Acad. Sci. USA 112, 5649.

Vikesland, P.J., Pruden, A., Alvarez, P.J.J., Aga, D.S., Bürgmann, H., Li, X-D., Manaia, C.M., Nambi, I., Wigginton, K., Zhang, T., Zhu, Y-G. (2017). Toward a comprehensive strategy to mitigate dissemination of environmental sources of antibiotic resistance. Environ. Sci. Technol. 51, 13061.

Wassenaar, T.M., Kist, M., and de Jong, A. (2007). Re-analysis of the risks attributed to ciprofloxacin-resistant Campylobacter jejuni infections. Int. J. Antimicrob. Agents. 30, 195.

WHO. (2014a). Briefing Note Antimicrobial Resistance: An Emerging Water, Sanitation and Hygiene Issue. Geneva: WHO.

WHO. (2014b). Critically Important Antimicrobials for Human Medicine, 5th revised list. Geneva: WHO.

WHO. (2015). Antimicrobial Resistance: Draft Global Action Plan on Antimicrobial Resistance. In Sixty-eight World Health Assembly. Geneva: WHO.

WHO. (2017). Who Guidelines On Use Of Medically Important Antimicrobials In Food-Producing Animals. Geneva: WHO.

Wright, G.D. (2007). The antibiotic resistome: The nexus of chemical and genetic diversity. Nat. Rev. Microbiol. 5, 175.

Wuijts, S., van den Berg, H.H., Miller, J., Abebe, L., Sobsey, M., Andremont, A., Medlicott, K.O., van Passel, M.W., and de Roda Husman, A.M. (2017). Towards a research agenda for water, sanitation and antimicrobial resistance. J. Water Health. 15, 175.

Xi, C., Zhang, Y., Marrs, C.F., Ye, W., Simon, C., Foxman, B., and Nriagu, J. (2009). Prevalence of antibiotic resistance in drinking water treatment and distribution systems. Appl. Environ. Microbiol. 75, 5714.

Zhang, T., and Li, B. (2011). Occurrence, Transformation, and Fate of Antibiotics in Municipal Wastewater Treatment Plants. Crit. Rev. Environ. Sci. Technol. 41, 951. 